\title{
Correlations between nailfold capillaroscopy findings and distinct clinical forms of juvenile dermatomyositis
}

\author{
Sigrid Covaci ${ }^{1,2}$, Oana-Maria Farkas ${ }^{3}$, Alexis-VirgilCochino ${ }^{1,3}$ \\ 1"Carol Davila" University of Medicine and Pharmacy, Bucharest, Romania \\ 2"Sf. Maria" University Hospital, Bucharest, Romania \\ 3"Alessandrescu-Rusescu" National Institute for Mother and Child Health, Bucharest, Romania
}

\begin{abstract}
Juvenile dermatomyositis is the most common idiopathic inflammatory myopathy in childhood. Nailfold capillaroscopy plays an important role in its assessment, being useful for diagnosis and follow-up. It is well documented that the morphological changes of nailfold capillaries reflect microvascular alterations presented in connective tissue diseases, including juvenile dermatomyositis. Several studies reported that the extent of microangiopathy detected by nailfold capillaroscopy examination offers a good correlation with disease activity and severity in juvenile dermatomyositis. The aim of this study was to assess the associations between distinct nailfold capillaries abnormalities and juvenile dermatomyositis clinical forms.
\end{abstract}

Keywords: nailfold capillaroscopy, children, juvenile dermatomyositis

\section{INTRODUCTION}

Juvenile dermatomyositis (JDM) is the most common idiopathic inflammatory myopathy in childhood. JDM is characterized by inflammation of the skin, muscles and sometimes other organ systems. A distinct rash and symmetrical weakness of proximal muscle groups are the key clinical features of JDM.

Bohan and Peter diagnostic criteria are still used for clinical practice and studies, although many clinicians choose not to perform electromyography or muscle biopsy, preferring non-invasive techniques, such as magnetic resonance imaging (MRI) (1). Assessment of children with definite or suspected JDM should include assessment of muscle weakness using a validated tool, such as the Childhood Myositis Assessment Scale (CMAS) and nailfold capillaries examination (2).

JDM varies in severity and progression, ranging from mild rash and muscle weakness to life-threatening forms, calcinosis or ulcerative disease. The clinical course of JDM correlates with the severity of the vascular disease (3).
Vasculopathy is considered central to JDM pathogenesis (4). Peripheral microangiopathy can be confirmed by nailfold capillaroscopy (NFC). The nailfold capillaries changes are almost always presented in JDM, sometimes visible to the naked eye $(5,6)$. The scleroderma-pattern, characterized by capillary dilation or giant capillaries and avascular areas, can be found in approximately 60\% patients with JDM (7). Compared with the changes found in systemic sclerosis, the microangiopathy of dermatomyositis shows more bushy capillaries with exuberant branching (8). The NFC findings at diagnosis cannot be used to predict the course of JDM but they can provide a sensitive parameter of chronic activity and damage, when the vascular abnormalities persist, and can help the clinician to consider continued and aggressive therapy (8). Previous studies documented that nailfold capillaries abnormalities correlate with more severe disease forms (ulcerative and chronic types), independently of disease activity or cutaneous abnormalities at the time of $\operatorname{NFC}(10,11,12)$. NFC findings correlated with skin disease activity (9) and cutaneous improvement is associated with 
the regeneration of capillaries (8). Spencer et al. demonstrated the relationship between NFC anomalies and muscle vasculopathy (10). Other authors found a strong association with the cutaneous but not with the musculoskeletal signs of JDM $(8,9)$. Heinrike et al. found that the loss of end row nailfold capillaries is better correlated to skin disease activity and less importantly to musculoskeletal involvement (14).

\section{MATERIALS AND METHODS}

Seventeen patients with JDM were studied. All patients fulfilled the Bohan and Peter classification criteria for definite JDM.

History and physical examination were taken focusing on the following: typical cutaneous changes (Gottron signs/papules, heliotrope rash, photosensitivity and periungual erythema), muscle weakness (history and CMAS score), calcinosis, ulcerations, respiratory (dyspnoea, dysphonia) and gastrointestinal symptoms (dysphagia). Laboratory assessment comprised complete blood count, erythrocyte sedimentation rate (ESR), serum levels of muscle enzymes [aspartate aminotransferase (AST), creatine kinase (CK), and lactic dehydrogenase (LDH)] and, in some cases specific/associated myositis autoantibodies.

NFC was performed in all patients on fingers 2 to 5 at both hands, using a stereomicroscope. The following parameters were assessed: integrity of the nailfold, capillaries' distribution, number and level of dropout, capillary enlargement (dilated and giant capillaries), atypical capillaries, such as crossed, bushy/branched and bizarre capillaries, micro-haemorrhages and predominant capillary pattern. Examination findings were considered abnormal if changes in density and morphology of nailfold capillaries were observed in two or more fingers.

\section{RESULTS}

This study included 17 JDM patients ( 5 boys and 12 girls) with ages between 2 and 16 years. All examinations were performed during the active phase of the disease, although first NFCs were at variable time from onset. All patients exhibited cutaneous alterations at the onset of the disease ( $3 / 17$ had serious cutaneous involvement, 10/17 moderate with typical cutaneous manifestations of JDM and 4/17 mild involvement represented by discreet heliotrope rash and/or Gottron signs). Muscle weakness was present in $15 / 17$ patients at the onset of disease $(5 / 17 \mathrm{had}$ important muscle weakness with CMAS 0-35, 10/17 had mild-moderate muscle weakness). Two patients exhibited amyopathic dermatomyositis.

Based on a complex examination, JDM patients were divided in 3 distinct clinical forms: classic, serious cutaneous involvement and severe.

Classic form. The 9 patients included in this group ( 6 girls and 3 boys) had characteristic dermatomyositis rash (heliotrope rash and Gottron signs/ papules over elbows, knees, metacarpophalangeal and interphalangeal joints) with concomitant minimum or moderate muscle involvement (weakness, increased serum levels of muscle enzymes and high ESR). One patient had a normal capillaroscopic pattern while 8 patients had abnormal nailfold capillaries. Six patients presented few enlarged capillaries, few micro-haemorrhages, relatively well-preserved capillary distribution, no evident loss of capillaries (Fig. 1). 6 of 9 patients presented branched capillaries (Fig. 2). Two patients presented giant capillaries, capillary haemorrhages, moderate loss of capillaries, mild disorganization of the capillary architecture (Fig. 3). One 8 years old girl with a more active and persistent cutaneous disease needed more complex treatment regimens and one 9 years old boy with long duration of untreated disease and progressive muscular involvement, finally developed calcinosis.

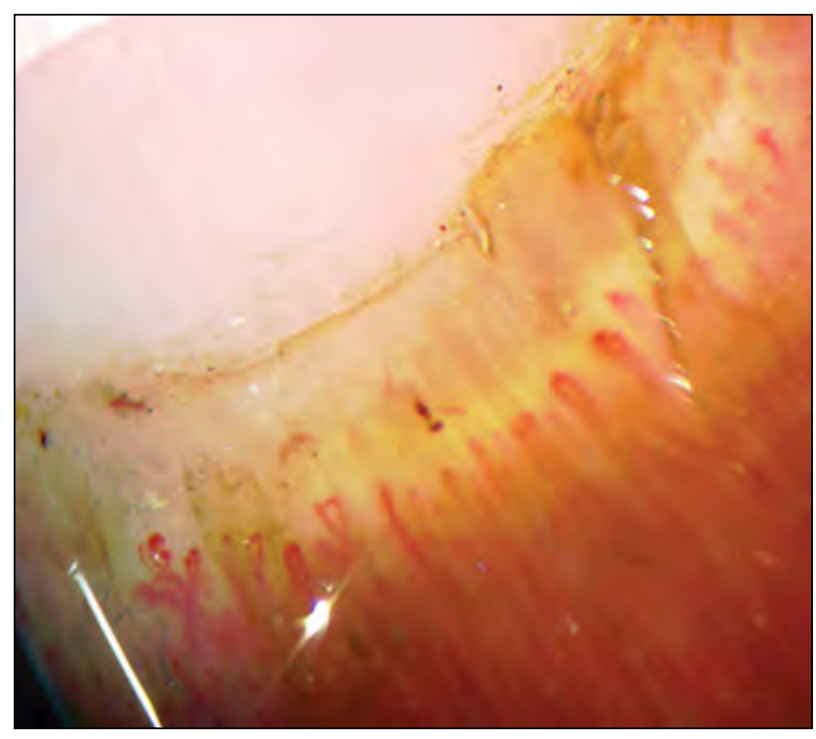

FIGURE 1. Few enlarged capillaries, one micro-haemorrhage, one branched capillary, relatively well-preserved capillary distribution, no evident loss of capillaries

The degree of capillary enlargement and the decrease in capillary density, as well as the presence of micro-haemorrhages and architectural disorganization, were closely related to disease activity, both muscle and skin involvement. 




FIGURE 2. Branched capillaries

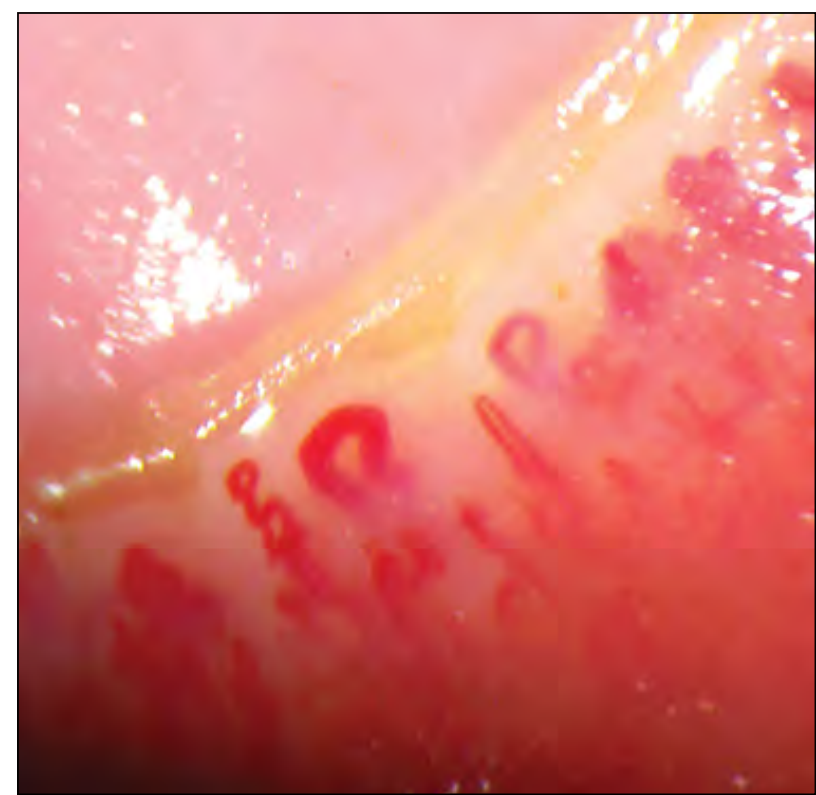

FIGURE 3. Giant capillaries, moderate loss of capillaries, mild disorganization of the capillary architecture

Serious cutaneous involvement form. The 3 patients ( 2 girls and 1 boy) included in this group had significant cutaneous findings: heliotrope rash, Gottron papules, periungual erythema, erythematous rash extended over the chest or limbs and photosensitivity. All 3 patients had a younger age at onset $(<5$ years old). Two of them had no clinical or laboratory evidence of muscle disease at disease onset, being diagnosed with amyopathic dermatomyositis. The other patient had moderate muscle involvement. In all cases, NFC examination revealed significant changes: loss of capillaries with avascular areas, enlargement of capillaries with giant capillaries; no bushy/branched capillaries were found (Fig. 4).

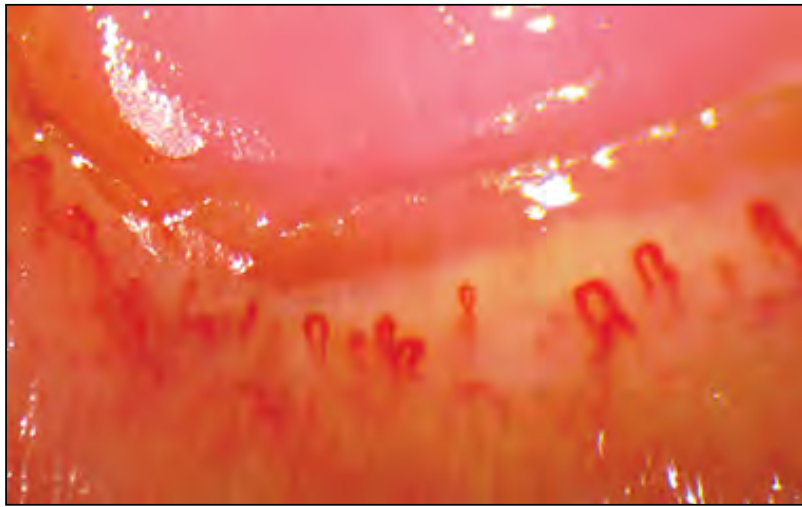

FIGURE 4. Loss of capillaries with avascular areas, giant capillaries

Severe form. Four of 5 patients in this group presented minimal cutaneous involvement (discreet heliotrope rash and/or Gottron papules) while a patient presented typical signs of JDM but with ulcerative lesions on elbows and knees. All patients presented important muscle weakness (CMAS 0-35) with increase in muscle enzymes and ESR. Three patients presented dysphonia, 2 of them with concomitant dysphagia and respiratory failure; one patient had macrophage activation syndrome shortly after the onset of the disease and another one lipodystrophy and ulcerative disease. NFC showed derangement of capillary architecture, capillary dropout and few isolated branched capillaries (Fig. 5). We found no data on NFC examination that could predict the course of disease.

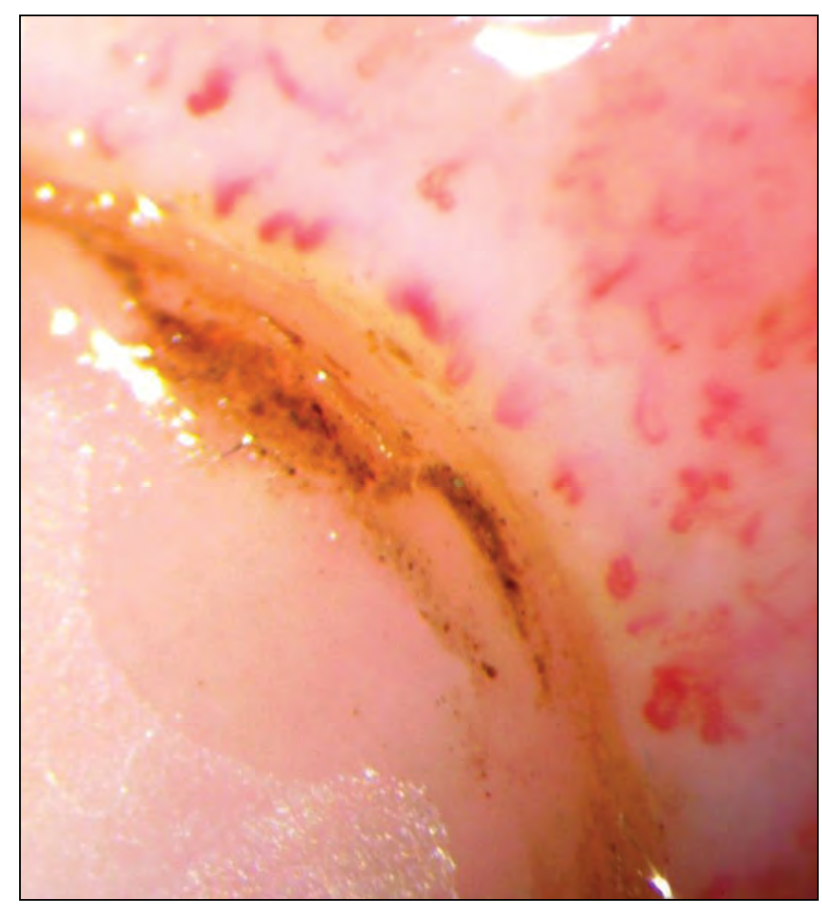

FIGURE 5. Derangement of capillary architecture, capillary dropout and a few bushes capillaries 
In all children in whom partial/total remission was achieved and a capillaroscopic follow-up was performed, we noticed nailfold capillaries regeneration and decreased numbers of enlarged and bushy capillaries.

\section{CONCLUSIONS}

Regardless of the disease form, nailfold capillaries abnormalities corelated with the disease activity. The degree of capillary enlargement and the decrease in capillary density, as well as the presence of micro-haemorrhages and architectural disorganization, are closely related to the degree of disease activity, in both muscle and skin manifestations.

\section{REFERENCES}

1. Brown VE, Pilkington CA, Feldman BM, Davidson JE (2006) An international consensus survey of the diagnostic criteria for juvenile dermatomyositis (JDM). Rheumatology 45(8): 990-993.

2. Martin N, Li, CK, \& Wedderburn LR (2012). Juvenile dermatomyositis: new insights and new treatment strategies. Therapeutic Advances in Musculoskeletal Disease, 41-50.

3. Crowe WE, Bove KE, Levinson JE, Hilton PK. Clinical and pathogenetic implications of histopathology in childhood polydermatomyositis. Arthritis Rheum. 1982;25:126-39.

4. Papadopoulou C, McCann LJ. (2018). The Vasculopathy of Juvenile Dermatomyositis. Front Pediatr. 2018 Oct 9;6:284. doi: 10.3389/fped.2018.00284

5. Dolezalova P, Young SP, Bacon PA, Southwood TR. Nailfold capillary microscopy in healthy children and in childhood rheumatic diseases: a prospective single blind observational study. Ann Rheum Dis2003;62:444-9.

6. Piotto DG, Len CA, Hilario MO, Terreri MT. Nailfold capillaroscopy in children and adolescents with rheumatic diseases. Rev Bras Reumatol 2012;52:722-32.

7. Carpentier $P$, Jeannoel $P$, Bost M, Franco A. Peri-ungual capillaroscopy in pediatric practice. Pediatrie 1988;43(2):165-9.

8. Smith RL, Sundberg J, Shamiyah E, Dyer A, Pachman LM. Skin involvement in juvenile dermatomyositis is associated with loss of end row nailfold capillary loops. J Rheumatol 2004;31(8):1644-9.
We found a close association between giant capillaries and serious cutaneous involvement and between capillary loss and severe form, significant cutaneous involvement and long duration of untreated disease.

In the group of patients with classical disease, the presence of bushy/branched capillaries was significantly higher compared to other clinical forms.

NFC performed during disease remission showed a tendency towards capillaries recovering numbers and morphology.

NFC plays an important role in the assessment of JDM (diagnose and disease activity monitoring), being an easily performed, non-traumatic, and lowcost method.

Conflict of interest: none declared Financial support: none declared

9. Christen-Zaech S, Seshadri R, Sundberg J, Paller AS, Pachman LM. Persistent association of nailfold capillaroscopy changes and skin involvement over thirty-six months with duration of untreated disease in patients with juvenile dermatomyositis. Arthr Rheum. (2008) 58:571-6. 10.1002/art.23299

10. Spencer-Green G, Crowe WE, Levinson JE. Nailfold capillary abnormalities and clinical outcome in childhood dermatomyositis. Arthritis Rheum 1982;25:954-8.

11. Nascif AK, Terreri MT, Len CA, Andrade LE, Hilario MO. Inflammatory myopathies in childhood: correlation between nailfold capillaroscopy findings and clinical and laboratory data. J Pediatr (Rio J) 2006;82(1):40-5.

12. Silver RM, Maricq HR. Childhood dermatomyositis: serial microvascular studies. Pediatrics 1989;83(2):278-83.

13. Spencer-Green G, Schlesinger M, Bove KE, et al. Nailfold capillary abnormalities in childhood rheumatic diseases, J Pediatrics, 1983, vol. 102 (pg. 341-6)

14. Heinrike Schmeling, Samantha Stephens, Cristina Goia, Cedric Manlhiot, Rayfel Schneider, Sanjeev Luthra, Elizabeth Stringer, Brian M. Feldman; Nailfold capillary density is importantly associated over time with muscle and skin disease activity in juvenile dermatomyositis, Rheumatology, Volume 50, Issue 5, 1 May 2011, Pages 885-893. 